\title{
Interventionelle Kardiologie
}

\author{
Christian Templin
}

Seit der ersten Ballondilatation eines Herzkranzgefässes durch Andreas Grüntzig im Jahr 1977 am Universitätsspital in Zürich hat sich die interventionelle Behandlung der koronaren Herzerkrankung sehr stark weiterentwickelt. Durch den Einsatz neuentwickelter Technologien ist eine zunehmend individualisierte Diagnostik und Therapie möglich, dennoch ergeben sich immer wieder neue Herausforderungen. So stellen zum Beispiel schwer verkalkte Läsionen komplexe Situationen dar, welche je nach Ausdehnung und Härtegrad gesonderter Therapien bedürfen, um ein perfektes Interventionsergebnis zu erzielen. Im Artikel von A. Patrascu et al. werden alt bewährte (u.a. Rotablation) und neue Therapieverfahren wie die Lithotripsie beschrieben. Der nächste Artikel von K. Mashayekhi et al. widmet sich den chronischen Koronararterienverschlüssen und der Frage nach der Evidenz bzw. welche Patienten von einer Rekanalisation profitieren. In den letzten Jahren ist es eher ruhig geworden um vaskuläre bioresorbierbare Scaffolds, welche noch vor einigen Jahren als faszinierendes neues Konzept vorgestellt wurden und das Potenzial besassen, Metallstents abzulösen. J. Gänsbacher et al. befassen sich mit der Frage, ob diese Therapie wirklich schon am Ende ist oder ob eine Renaissance zu erwarten ist. Im Zeitalter der zunehmenden Individualisierung erscheint die intrakoronare Bildgebung einen besonderen Stellenwert einzunehmen. Im Beitrag von S. Bär et al. werden unterschiedliche Imagingverfahren vorgestellt und deren Möglichkeiten in Diagnostik und Therapieplanung bzw. -kontrolle erläutert. Der koronaren Mikrozirkulation kommt insbesondere beim Ausschluss von epikardialen Koronarstenosen, insbesondere bei Frauen eine zunehmende Bedeutung zu. So zeigen ca. $10 \%$ der Patienten mit akutem Myokardinfarkt keine obstruktiven Läsionen (Myokardinfarkt mit nichtobstruktiven Koronararterien, MINOCA). Auch das Takotsubo Syndrom ist durch eine Mikrozirkulationsstörung gekennzeichnet. Im Artikel von A. Candreva et al. wird auf dieses und Krankheitsbilder aus der Gruppe der MINOCA eingegangen und der Stellenwert der invasiven Messung der Mikrozirkulation beschrieben. Ebenso stellen spontane Koronararteriendissektionen (SCAD) eine zunehmend di- agnostizierte Ätiologie des akuten Koronarsyndroms und eine wichtige Ursache des Myokardinfarktes bei Frauen dar. Sie wird inzwischen als Ursache von $4 \%$ aller und $25 \%$ der akuten Koronarsyndrome bei Frauen unter 50 Jahren angenommen und ist die häufigste Ursache des Myokardinfarktes in der Schwangerschaft. Mit dieser Thematik befasst sich der Artikel von M. Würdinger et al., welcher einen Überblick über den aktuellen Wissensstand bietet. Abgerundet wird das koronare Themengebiet mit dem Artikel von S-H. Kim et al., welcher unter Berücksichtigung der aktuellen ESC-Leitlinien und des ISCHEMIA Trials das diagnostische und therapeutische Vorgehen bei Patienten mit chronischem Koronarsyndrom diskutiert.

Auch im Bereich struktureller Herzinterventionen hat sich in den letzten Jahren viel getan. So hat sich die Transkatheter-Aortenklappenimplantation (TAVI) als Standardverfahren für die Behandlung schwerer Aortenklappenstenosen etabliert und bereits einen grösseren Stellenwert als der chirurgische Aortenklappenersatz eingenommen. J. Michel et al. beschreiben in ihrem Artikel die aktuell bestehenden Herausforderungen und Behandlungsentscheidungen im Rahmen einer TAVI wie Valve-in-Valve-Verfahren, die bikuspiden Aortenklappe und die Auswahl der antithrombotischen Therapie nach TAVI.

Ich bedanke mich bei allen Autoren und hoffe sehr, dass die aktuelle Ausgabe der «Therapeutischen Umschau» Ihnen eine praxisnahe Lektüre zu aktuellen Themen der interventionellen Kardiologie bietet.

Prof. Dr. med. Dr. rer. nat. Christian Templin

Leitender Arzt Kardiologie

Leiter Andreas-Grüntzig-Herzkatheterlabore

Universitätsspital Zürich

Universitäres Herzzentrum Zürich

Rämistrasse 100

8091 Zürich

christian.templin@usz.ch 